\title{
A Psicologia na Atenção Básica: Possibilidades de Intervenção na Promoção e Prevenção à Saúde
}

\section{Psychology in Primary Health Care: Possibilities for Intervening in Health Promotion and Prevention}

\author{
La Psicología en la Atención Básica: Posibilidades de Intervención en la Promoción y \\ Prevención de la Salud
}

\author{
Natália Batista Rosa ${ }^{1}$ \\ Ana Cláudia Barbosa da Silva-Roosli \\ Universidade Estadual de Londrina (UEL)
}

\begin{abstract}
Resumo
Este relato de experiência descreve intervenções realizadas por uma psicóloga vinculada a um Programa de Residência Multiprofissional em Saúde da Família, desenvolvidas numa Unidade Básica de Saúde (UBS). Objetiva-se analisá-las sob a ótica das contribuições da Psicologia para ações de saúde de caráter coletivo na Atenção Básica. As intervenções foram registradas e submetidas à análise qualitativa. Na participação do planejamento e execução do apoio matricial, das articulações intersetoriais, das abordagens comunitárias e atendimentos compartilhados, concedeu-se atenção à dimensão gestionária do trabalho. Assim, incorporaram-se os saberes da prática dos profissionais à construção das ações de saúde. Os resultados indicam o fortalecimento da capacidade normativa de trabalhadores e da compreensão dos singulares modos de vida dos usuários, bem como a potencialização do trabalho em equipe. Conclui-se que a valorização do ponto de vista dos trabalhadores sobre o trabalho colaborou para o aprimoramento da oferta de ações de promoção e prevenção à saúde.

Palavras-chave: atuação da Psicologia, saúde da família, Núcleo de Apoio à Saúde da Família, dimensão gestionária do trabalho, apoio matricial
\end{abstract}

\begin{abstract}
This report describes interventions performed by a psychologist linked to a Multiprofessional Residency Program in Family Health Care, which were developed in a Basic Health Unit (UBS). This paper aims to analyze these interventions from the perspective of the Psychology contributions to collective health actions on the primary care level. These interventions were recorded and submitted to qualitative analysis. Attention was given to the managerial dimension of work when addressing the participation in planning and executing the matrix support, intersectoral articulations, community approaches and shared services. Thus, the professionals' practice knowledge was incorporated into the construction of health actions. The results indicate the strengthening of the regulatory capacity of workers and understanding the unique ways of life of users, as well as the enhancement of teamwork. It is concluded that the valorization of the workers' point of view on their work contributed to enhance the health promotion and prevention actions offer.

Keywords: Psychology actions, family health, Support Center for Family Health, managerial dimension of work, matrix support

\section{Resumen}

Este relato de experiencia presenta intervenciones realizadas por una psicóloga vinculada a un Programa de Residencia Multiprofesional en Salud de la Familia desarrolladas en una Unidad Primaria de Salud (UBS). El objetivo es analizarlas bajo la óptica de las contribuciones de la psicología para acciones de la salud de carácter colectivo en la Atención Primaria. Las intervenciones fueron registradas y sometidas a análisis cualitativo. En la participación del planeamiento y ejecución del apoyo matricial, de las articulaciones intersectoriales, de los abordajes comunitarios y atendimientos compartidos, se concedió atención a la dimensión de gestión del trabajo. Por lo tanto, se incorporó los saberes de la práctica de los profesionales a la construcción de las acciones de la salud. Los resultados indican el fortalecimiento de la capacidad normativa de trabajadores y de la comprensión de los singulares modos de vida de los usuarios, como también la potenciación del trabajo en equipo. Se concluye que la valoración del punto de vista de los trabajadores sobre el trabajo ha colaborado para
\end{abstract}

\footnotetext{
${ }^{1}$ Endereço de contato: Rua da Lapa, 102, Edifício Vale do Sol, Apto. 106, Parque Bela Vista, CEP: 86015-050, Londrina, PR, Brasil. Contato:(43)99627-3010. Email: nataliab.rosa@hotmail.com
} 
el perfeccionamiento de la oferta de acciones de promoción y prevención de la salud.

Palabras clave: actuación de la Psicología, salud de la familia, Núcleo de Apoyo a la Salud de la Familia, dimensión de gestión del trabajo, apoyo matricial

\section{Introdução}

A inserção da Psicologia na assistência pública à saúde, enquanto profissão, remonta a períodos anteriores à sua regulamentação no Brasil, ocorrida em 1962; especialmente na década de 1950, com ações pontuais na área hospitalar e de atenção materno-infantil (Cezar, Rodrigues, \& Arpini, 2015). Sua consolidação dá-se durante as décadas de 1970 e 1980, concomitantemente ao movimento da Reforma Sanitária que culminou na implantação do Sistema Único de Saúde (SUS).

Uma breve revisão histórica revela que, principalmente até a década de 1980, a inserção da Psicologia no campo da saúde era marcada pela prática clínica caracterizada por um viés privatista, elitista e apartado do contexto social do país. A atuação era realizada em consultórios particulares (dirigida a uma clientela derivada de classes abastadas), em hospitais e ambulatórios de saúde mental (orientada pelo enfoque, então dominante, de internação e medicação) (Bock, 1999; Spink, 2003).

Nas últimas décadas, nota-se importante participação da Psicologia no que concerne à garantia do direito à atenção integral à saúde, através do trabalho cotidiano no SUS. A partir da pesquisa sobre a profissão do psicólogo no Brasil, Macedo, Heloani, e Cassiolato (2010) traçaram o perfil do profissional psicólogo assalariado, caracterizando os locais, atividades e condições de trabalho conforme o setor produtivo - público, privado e terceiro setor. Ao comparar a inserção dos psicólogos entre os diferentes setores, esses autores identificaram que o setor público é o maior empregador de psicólogos; destacando-se maior inserção de psicólogos nos setores de administração municipal $(45,9 \%)$, se comparado aos setores de administração estadual (31,1\%) e federal (23\%).

Tal diferença estaria relacionada ao crescimento da inserção do psicólogo nas equipes de saúde, sob responsabilidade dos municípios; isso os levou a concluir que a maioria dos psicólogos empregados no setor público trabalham na área da saúde (Macedo et al., 2010).

Entre as diversas possibilidades de inserção da Psicologia na assistência pública à saúde, este trabalho tem como foco a Atenção Básica (AB), primeiro nível de assistência do SUS. A $A B$ delimita-se como um conjunto de ações de saúde no âmbito individual e coletivo que abrangem a promoção e proteção da saúde, prevenção de agravos, diagnóstico, tratamento, reabilitação e manutenção da saúde.

Em 2006, o Ministério da Saúde (MS) publicou a Política Nacional de Atenção Básica (PNAB), estabeleceu a revisão de diretrizes e normas para a organização da $A B$ e alça a Saúde da Família (SF) como "estratégia nacional" e não mais como "programa" (Brasil, 2006, 2011). Assim, a SF foi apresentada como estratégia para reverter o modelo de assistência vigente e fortalecer a $A B$ como eixo de orientação da assistência à saúde, colocando em sintonia os princípios preconizados pelo SUS.

Registra-se que a entrada oficial da Psicologia nesse nível de assistência ocorreu com sua integração às equipes do Núcleo de Apoio à Saúde da Família (NASF). Criado por meio da Portaria n. 154 de 24 de janeiro de 2008 (Brasil, 2008), o NASF expressa o engajamento do MS para o fortalecimento da $A B$. 
A fim de atender diferentes populações e realidades do Brasil, o NASF é uma nova modelagem de equipe, composta por profissionais de diversas especialidades, incluindo a Psicologia, que atuam em parceria com as equipes de Saúde da Família (eSF), "compartilhando práticas e saberes em saúde com as equipes de referência apoiadas, buscando auxiliá-las no manejo ou resolução de problemas clínicos e sanitários, bem como agregando práticas, na atenção básica, que ampliem o seu escopo de ofertas" (Brasil, 2014, p. 19).

Por meio do NASF, almeja-se organizar e estruturar a atuação de diversos especialistas na AB-psicólogos, fisioterapeutas, nutricionistas, farmacêuticos, educadores físicos, terapeutas ocupacionais, assistentes sociais, entre outros.

No mesmo intuito de fortalecer a AB, criou-se também, em forma da lei n. 11.129/2005, o Programa da Residência Multiprofissional em Saúde da Família (RMSF). Esse Programa constitui-se numa modalidade de educação em serviço e representa mais uma iniciativa para o fortalecimento da AB (Brasil, 2012). Seu objetivo é favorecer a inserção qualificada dos jovens profissionais da saúde no mercado de trabalho, particularmente para a construção do SUS.

Em Londrina, no Estado do Paraná, a proposta da RMSF surgiu pela parceria entre a Universidade e a Secretaria Municipal de Saúde (SMS). Organizou-se visando colaborar na implementação da Estratégia Saúde da Família (ESF) na cidade, além de formar profissionais capacitados para atuarem na $A B$ com enfoque multiprofissional, fortalecendo o desenvolvimento do trabalho em equipe de maneira interdisciplinar (Domingos, Nunes, \& Carvalho, 2015).

Criado em 2007, através de convênios com o MS, o curso de Especialização em Saúde da Família na modalidade de Residência Multiprofissional em Saúde da Família (RMSF) institucionalizou-se como projeto da Universidade em 2010. Com duração de dois anos, adota como metodologia a integração entre teoria e prática e o desenvolvimento de conteúdos que permitam sustentar práticas articuladas na AB.

Na configuração atual, as práticas assistenciais dos residentes de diversas especialidades são orientadas pelas diretrizes do NASF. A organização do trabalho pactuada com a SMS viabilizou a atuação dos residentes, formando duas equipes NASF responsáveis pelo apoio especializado a duas Unidades Básicas de Saúde (UBS), cada qual com apenas duas eSF. Entende-se que o formato de trabalho pactuado colaborou para a superação de fatores limitantes e problemáticos comuns na implantação do NASF (Brasil, 2014), pois favoreceu a construção de vínculos mais sólidos com os trabalhadores da UBS, bem como a aproximação da realidade territorial de determinado serviço de saúde (cada equipe atrelou-se a apenas uma UBS); isso difere do formato de trabalho prescrito às equipes NASF, que precisam conciliar diferentes realidades por vincularem-se a mais de uma UBS.

No que que diz respeito à inserção da Psicologia na AB, Dimenstein $(1998,2006)$ sublinha a reduzida quantidade de pesquisas sobre o trabalho do psicólogo nesse nível de assistência, tanto nacionais quanto locais, indicam a pertinência de reflexão sobre a atuação nesse campo específico. Azevedo e Kind (2013) explicitam que a produção bibliográfica acerca do NASF ainda é incipiente, o que impulsiona a busca de documentos oficiais, federais e municipais sobre o tema.

Pautando-se por essas considerações, neste relato procurou-se cotejar o trabalho da psicóloga na UBS, isoladamente ou em parceria com outros profissionais, considerando-se 
as diretrizes da $A B$ e do NASF e incorporando à análise as contribuições do conceito de saúde apresentado por Georges Canguilhem e aportes teóricos da Psicologia do Trabalho e Organizacional.

Para Canguilhem, pensar num conceito de saúde é ter referências sobre a vida e formas de intervenção sobre ela. Segundo Caponi (1997), Canguilhem introduz uma importante inversão nos estudos sobre a saúde, que deixa de ser definida por oposição à doença. A saúde passa a ser definida como a capacidade de criar novas normas, valores e modos de viver frente às infidelidades, variações e imprevistos inerentes à própria vida. Ou seja, a saúde identifica-se com a capacidade humana de criar normas e valores, o que Canguilhem denominou de normatividade vital.

Essa concepção admite, no interior do conceito de saúde, a capacidade de adaptação. Contudo, ao afirmar a saúde como a capacidade de criar novas normas de vida, extrapola a ideia de associar a saúde ao ajuste ou adaptação a um meio.

A saúde é pensada "em termos de margem de tolerância (ou de segurança), que cada um possui para enfrentar e superar as infidelidades do meio" (Caponi, 1997, p. 291) e como abertura ao risco, ou seja, a capacidade de tolerar, enfrentar e superar as infidelidades do meio. Dessa forma, a saúde é um produto do debate permanente com o meio e não implica somente segurança contra os riscos, mas a capacidade de enfrentá-los, corrigindo a margem de segurança.

Explorar as contribuições de Canguilhem sugere que as ações de saúde não devem apenas ordenar uma série de condutas que gerem bem-estar aos usuários ou evitem riscos. Devem, também, dar condições de escolha e criação aos indivíduos, colaborar para ampliar as margens de segurança e a abertura ao risco o máximo possível.

Nessa direção, o trabalho da psicóloga objetivou colaborar para a criação de normas de cuidado que fortalecessem uma postura mais compreensiva do que prescritiva das normas de vida dos usuários adscritos à área de abrangência das eSF em questão.

Embora a profissional tenha realizado atendimentos clínicos individuais, para este estudo tomou-se como foco as ações de caráter coletivo, indicadas como difíceis de serem concretizadas por distanciarem-se da atuação clínica tradicional em Psicologia, como atestam diversos estudos (Amaral, Gonçalves, \& Serpa, 2012; Azevedo \& Kind, 2013; Cezar et al., 2015; Clemente et al., 2008).

Possuindo como alicerce o trabalho em equipe, problematizar as ações coletivas de saúde conduziu à integração de contribuições de conhecimentos disponibilizados por diversas áreas da Psicologia, entre os quais se destaca a Psicologia do Trabalho e Organizacional. Nesse caso, a inserção no trabalho das eSF procurou, constantemente, mobilizar um olhar sob o ponto de vista da atividade de trabalho, pautando-se no constructo teórico atividade desenvolvido por diversas "teorias da atividade" (Athayde \& Zambroni-de-Souza, 2015).

Nas intervenções da psicóloga, adotar esse ponto de vista implicou incorporar os saberes da prática dos profissionais de saúde e a dimensão gestionária do trabalho na construção das ações de saúde. No processo de articulação do trabalho junto às eSF, deu-se atenção à gestão das infidelidades do meio de trabalho realizada por todos os trabalhadores, independente da formação profissional ou cargo ocupado. Gestão do trabalho aqui compreendida como "fazer escolhas, 'renormatizar', criar novas regras em situação. Gerir sendo entendido em um sentido complexo, em uma dimensão profissional, econômica, intersubjetiva e pessoal“ 
(Oliveira \& Brito, 2011, p. 270). Portanto a compreensão de gestão adotada considera-a como dimensão pertencente ao processo de trabalho de todos os profissionais de saúde, não se restringindo somente àqueles que ocupam um cargo formal, como o coordenador da UBS.

\section{Metodologia}

Este relato de experiência caracteriza-se como um estudo descritivo e qualitativo. As intervenções da psicóloga, realizadas na UBS no âmbito de formação do Programa da Residência Multiprofissional em Saúde da Família (RMSF) entre os anos 2014 e 2016, foram registradas em diário de campo.

A análise das intervenções orientou-se tanto pela leitura da dimensão prescrita do trabaIho, acessada pelos documentos oficiais do MS como Política Nacional de Atenção Básica e Cadernos de Atenção Básica, quanto pelas contribuições do conceito de saúde de Georges Canguilhem e aportes teóricos da Psicologia do Trabalho e Organizacional, apresentados acima.

A opção pelas ações de caráter coletivo ancorou-se em constatações e interrogações das demandas direcionadas à psicóloga na UBS: frequentemente eram privilegiadas demandas julgadas previamente como pertencentes, exclusivamente, ao campo da Saúde Mental. Originadas de diversos atores- demais residentes, profissionais da UBS e das eSF, representantes da gerência municipal e dos próprios usuários-, era recorrente a demanda por atendimentos clínicos individuais, focada na abertura de horários na agenda da psicóloga.

Tal aspecto também é citado pelo MS (Brasil, 2014) como um fator limitante das possibilidades de ação do NASF:

a predominância da lógica de atenção centrada na doença e em procedimentos curativos muitas vezes ocasiona pressão para que o Nasf trabalhe em uma lógica ambulatorial, centrada apenas na dimensão assistencial do apoio matricial. Essa pressão pode ser realizada ora pela população - que tem a expectativa de ser atendida individualmente -, ora pela própria equipe de $A B$ - que, muitas vezes, espera do Nasf apenas suporte para atendimentos clínicos diretos -, ora pela gestão - que pode monitorar ou cobrar apenas a produção quantitativa de atendimentos, ora pelos profissionais do Nasf (p. 39).

Esse fato conduziu ao questionamento sobre as possibilidades do processo de trabalho do psicólogo na $A B$ e à reflexão quanto aos efeitos de reduzi-lo ao atendimento clínico individual. Essa questão também gerou inquietações em Nepomuceno (2009), para o qual:

o modo como o Nasf está preconizado, na portaria que o institui, é a Psicologia colocada somente dentro do marco da Saúde Mental. Tal inquietação se dá pelo fato de que a Psicologia, na Saúde Pública, já tem essa história de trabalhos vinculados a um tipo de prática clínica reducionista, ligada a uma visão limitada à cura de psicopatologias e à reprodução de práticas de atenção ambulatorial de atendimento individual, de enfoque psicopatologizante. As contribuições da Psicologia à ESF e ao SUS são bem mais amplas e transcendem a dimensão curativista (p. 55).

Essas constatações conciliadas à atenção aos modos de vida dos usuários da UBS foram 
problematizadas e analisadas pelo núcleo de Psicologia da RMSF durante a orientação das intervenções da psicóloga.

\section{Resultados e Discussão}

\section{A Psicologia e as Contribuições ao Apoio Matricial: Promoção e Prevenção à Saúde em Perspectiva}

Conforme as diretrizes do MS (Brasil, 2010, 2014), o processo de trabalho do NASF deve ser desenvolvido por meio do Apoio Matricial a partir da criação de espaços coletivos para discussões e planejamento. Tais espaços podem ser estruturados no formato de reuniões regulares realizadas entre as equipes e os profissionais do NASF ou em momentos informais, nos quais deverão ser organizadas e estruturadas: ações clínicas compartilhadas; intervenções específicas do profissional do NASF com os usuários e/ou famílias; além de abordagens compartilhadas no território.

O conceito de Apoio Matricial é definido como uma retaguarda especializada assegurada às equipes e profissionais encarregados da atenção aos problemas de saúde dos usuários dos serviços, de forma interativa e orientada à singularidade dos casos (Campos \& Cunha, 2011; Campos \& Domitti, 2007; Melo et al., 2012). Apresenta-se em duas dimensões- suporte assistencial e técnico-pedagógico- que necessitam estar associadas nos diversos momentos de atuação. A dimensão assistencial deve produzir ação clínica direta com os usuários, enquanto a técnico-pedagógica produzirá ação de apoio educativo junto às equipes de referência, isto é, as eSF.

Por serem portadores de conhecimentos distintos daqueles concernentes às eSF, os profissionais do NASF contribuem com estas por meio do compartilhamento de saberes técnico-científicos, visando a intervenções que aumentem sua capacidade resolutiva, pelo fato de tais equipes serem consideradas responsáveis primariamente pelo atendimento dos casos.

A população adscrita à área de abrangência da UBS era dividida entre duas eSF (equipes A e B), as quais eram apoiadas pelos diversos profissionais residentes da RMSF. Eram realizadas reuniões semanais de matriciamento com cada uma das equipes, objetivando o desenvolvimento do Apoio Matricial.

As reuniões duravam cerca de duas horas e, nesses espaços, os casos específicos bem como as ações realizadas pelos profissionais no decorrer da semana e dos meses eram apresentados, analisados e refletidos de forma coletiva, propiciando orientações, trocas de saberes e, quando necessário, elaboração de planos de cuidado compartilhados entre os profissionais de cada equipe- em casos mais complexos, o instrumento utilizado era o Projeto Terapêutico Singular (PTS).

Conjuntamente, os profissionais das equipes e a psicóloga utilizaram essas ocasiões para desenvolver o matriciamento com relação aos usuários, contextualizando o processo saúde-doença de modo a ampliar a compreensão de suas histórias enquanto sujeitos singulares, atravessados por complexas condições socioeconômicas, culturais e construções subjetivas. Ao final das discussões eram pactuadas as visitas domiciliares a serem feitas no dia, ou no decorrer da semana, com base na relevância da contribuição de cada profissional. 
Entre os casos que mais demandaram o Apoio Matricial da área de Psicologia, estavam os relacionados a usuários com diagnósticos psiquiátricos. Nessas situações, foi possível trabalhar aspectos referentes ao tratamento e manejo em saúde mental com as famílias e profissionais das equipes. Associados a esses casos, estavam aqueles envolvendo crianças, mulheres e idosos em situação de violência intrafamiliar, negligência, abuso sexual e psicológico.

Para fins de demonstração das contribuições da psicóloga no Apoio Matricial, selecionou-se a seguinte situação: durante uma reunião de matriciamento, a estagiária de enfermagem apresentou a história de um usuário com diagnóstico de esquizofrenia que se negava a fazer uso das medicações psicotrópicas prescritas. A princípio, os profissionais da equipe demandaram da psicóloga uma abordagem do usuário e sua mãe (cuidadora idosa com limitações auditivas), por avaliarem o caso pertencente aos cuidados da Atenção Psicossocial.

Perante esse caso, iniciou-se a discussão a respeito do acompanhamento de usuários com transtornos mentais, refletindo acerca das condutas possíveis na AB; além da problematização quanto a concepções equivocadas e receios existentes em torno das doenças consideradas psiquiátricas - por vezes, os profissionais negavam-se a realizar atendimentos a usuários com esse perfil.

As residentes de Psicologia e Serviço Social acompanharam a família de forma contínua por meio de visitas domiciliares (semanais, inicialmente, depois mensais e, por fim, a cada 45 dias); criaram vínculo com a mãe e, posteriormente, com seu filho.

Ao identificar as características do contexto dessa família, realizou-se um processo de sensibilização da equipe: a ACS do território passou a realizar visitas; o enfermeiro e o médico realizaram algumas abordagens para orientação e solicitação de exames, reconhecendo os sintomas e queixas apresentados anteriormente pelo usuário como necessidades de saúde. No decorrer do processo, as reuniões de Apoio Matricial propiciaram a pactuação de estratégias compartilhadas, como a inserção da família no acompanhamento domiciliar feito regularmente pela eSF, com a frequência de visitas domiciliares a cada 45 dias.

A partir do acompanhamento sistemático, a cuidadora idosa começou a frequentar a UBS para cuidar da própria saúde, e o filho retornou ao Centro de Atenção Psicossocial (CAPS) para reavaliação, aderindo ao tratamento medicamentoso. O fortalecimento dos vínculos entre a família e os profissionais, os quais realizaram as abordagens promovendo a oferta de acolhimento, escuta terapêutica e apoio psicossocial, ofereceram indícios de que as orientações quanto aos cuidados de saúde tornaram-se significativas para essa família.

A postura adotada pela psicóloga junto à eSF foi a de questionar e colocar em debate os diferentes pontos de vista, julgamentos e decisões para a definição (coletiva e compartilhada) das ações de saúde. Acredita-se que essa forma de direcionamento do caso colaborou para que as orientações, quanto aos cuidados de saúde da família, tornassem-se dotadas de sentido para tais profissionais.

$\mathrm{Na}$ experiência com o Apoio Matricial, foi possível analisar que, conforme proposto pelas diretrizes do NASF, para serem efetivos tanto o planejamento, quanto a organização e execução das ações de saúde pelas equipes, seria necessário o compartilhamento de saberes e responsabilidades, além da participação ativa dos profissionais do NASF e das eSF no processo. 
Constatou-se que as ações de Apoio Matricial estiveram interligadas às de promoção, prevenção e reabilitação da saúde durante a atuação da psicóloga, sendo essenciais para o acompanhamento dos casos mais complexos (apoio assistencial) e capacitação dos diversos atores implicados na produção do cuidado (apoio técnico-pedagógico às equipes multiprofissionais).

\section{As Intervenções da Psicologia sob o Enfoque da Promoção e Prevenção à Saúde}

De acordo com Medina et al. (2014), poucos estudos no Brasil problematizaram ou avaliaram a natureza e efetividade das ações de promoção à saúde desenvolvidas na AB. O que evidencia a incipiência quanto à reflexão e conceituação precisas do tema, além disso, indica a pouca clareza dos profissionais que atuam nesse nível de assistência.

Sícoli e Nascimento (2003) referem que, apesar de ainda persistirem controvérsias na definição do conceito, além de confusões quanto a seus limites com a prevenção, muitos autores vêm procurando desenvolver, elucidar e difundir o discurso da promoção. Principalmente considerando-se o contexto brasileiro atual, no qual se evidencia a busca de modelos de atenção à saúde que extrapolem o modelo assistencial hospitalocêntrico.

Assim, conforme os autores, pode-se dizer que a promoção destaca-se ao resgatar o ponto de vista da saúde enquanto produção social, visando aprimorar políticas públicas bem como ações coletivas que ultrapassem até mesmo o enfoque do risco (campo da prevenção).

Segundo Czeresnia (2003) "as estratégias de promoção enfatizam a transformação das condições de vida e de trabalho que conformam a estrutura subjacente aos problemas de saúde" (p. 7) e demandam uma abordagem intersetorial. Para a autora, a promoção da saúde refere-se a medidas que não se restringem a uma determinada doença ou agravo, mas servem para aumentar a saúde e o bem-estar gerais.

No que se refere à articulação intersetorial, o NASF é apontado como uma alternativa para superar a fragmentação do modelo assistencial, estabelecendo conexões entre profissionais e equipes de diferentes serviços - incluindo os de outros setores, como a Assistência Social e a Educação -, potencializando a construção de redes e fluxos assistenciais no território de atuação dessas equipes (Brasil, 2014).

Nessa direção, foram promovidas articulações com outros setores na forma de reuniões intersetoriais realizadas com profissionais representantes de políticas públicas que acompanhavam as famílias adscritas à área de abrangência da UBS, como: Assistência Social, Educação, Organizações Não Governamentais (ONGs) e Conselho Tutelar. Tais reuniões, para discussão de casos e compartilhamento do cuidado, foram mobilizadas a partir da observação e análise de demandas verificadas no território junto aos usuários e famílias assistidos, além das solicitações de atendimento psicológico advindas dos respectivos serviços.

A participação em reuniões intersetoriais de frequência periódica ocorreu devido ao acompanhamento de casos de risco (famílias em situação de vulnerabilidade social) e foi compartilhada com os demais profissionais do NASF e das eSF, sob coordenação do Centro de Referência em Assistência Social (CRAS) da região.

As articulações intersetoriais resultaram no desenvolvimento de estudos de caso, visitas domiciliares compartilhadas, construção de fluxos de encaminhamento e atendimento a usuários com direitos violados, bem como a pactuação de abordagens no território, como 
oficinas e palestras sobre os seguintes temas: autocuidado e hábitos alimentares saudáveis junto a crianças de escolas municipais e projetos sociais; saúde sexual e reprodutiva com adolescentes e jovens de colégios estaduais; e, tabagismo associado à saúde bucal para trabalhadores de empresas da região.

De acordo com Spink, Menegon, Gamba, e Lisboa (2007), a prática dessas atividades aponta formas de atuação mais abrangentes para o psicólogo, tendo como objeto os sujeitos nas suas relações, inseridos em um território. Ou seja, não restritos aos muros institucionais e ao enfoque patológico, buscando atingir a população quanto aos determinantes e condicionantes sociais de saúde. Observou-se certo desconhecimento sobre as contribuições da Psicologia para a compreensão do processo saúde-doença, bem como sua função no contexto da AB. Assim, os espaços de abordagem coletiva- grupos com usuários e reuniões intersetoriais - oportunizaram a divulgação e fortalecimento do trabalho da psicóloga no território junto aos usuários assistidos e aos serviços vinculados. Pode-se dizer que as intervenções com grupos e reuniões intersetoriais constituíram-se como importantes espaços de troca de saberes, também voltados ao esclarecimento das demandas e dúvidas quanto ao manejo da psicóloga.

O fato de reconhecer outras ordens de determinação no processo saúde-doença propiciou intervenções menos fragmentadas, o que tem sido uma dificuldade para os psicólogos na área da saúde devido à tendência de conceber isoladamente as problemáticas, descaracterizando o sentido político social da atuação (Andrade \& Araújo, 2003; Dimenstein \& Macedo, 2007).

No interior da UBS, os constantes diálogos com os profissionais das eSF colaboraram para a abertura de uma frente de ação atenta a populações em situação de vulnerabilidade social, ou seja, atravessadas por questões de pobreza, drogadição, exploração sexual e financeira, violência e tráfico (Ayres, França Júnior, Calazans, \& Saletti Filho, 2003). Em regiões geograficamente mais afastadas da UBS, existiam poucas ações de saúde ofertadas para tal população. Perante essa constatação, sensibilizou-se os profissionais acerca de graves situações recorrentes e, progressivamente, essa população foi incorporada ao acompanhamento longitudinal do território realizado pelas eSF e NASF.

Já as ações preventivas são definidas como intervenções orientadas a evitar o surgimento de doenças específicas, de modo a reduzir sua incidência e prevalência nas populações (Czeresnia, 2003). Freitas (2003) afirma que as estratégias de prevenção às doenças direcionam-se, de forma diretiva e persuasiva, principalmente aos grupos de alto risco na população, isto é, acometidos por patologias específicas. Assim, constituem programas focalizando fundamentalmente tópicos individuais e de grupos.

Na perspectiva da prevenção à saúde, o trabalho da psicóloga inseriu-se no acompanhamento ao pré-natal; discussão de casos de crianças e adolescentes no CRAS, Centro de Referência Especializado em Assistência Social (CREAS), CAPS, escolas e projetos sociais; além de visitas domiciliares para orientação de famílias com conflitos. Tais ações apresentaram caráter multiprofissional e interdisciplinar.

Destaca-se a intervenção referente ao Programa Saúde na Escola (PSE), com vistas à prevenção da saúde. O PSE configura-se como política intersetorial entre educação e saúde, compreendendo a escola enquanto espaço para práticas de promoção de saúde e prevenção 
de agravos e doenças, por meio de ações compartilhadas entre profissionais de ambos os setores; nesse sentido, atribui-se ao NASF o apoio às equipes de saúde e educação a partir de demandas específicas, em sintonia com outros articuladores do PSE (Brasil, 2014).

Realizou-se uma oficina com as professoras e diretora de uma creche do território que atendia crianças de zero a dois anos. Conduzida junto com a educadora física residente e com o enfermeiro da eSF, teve como objetivos a sensibilização e capacitação das profissionais sobre o tema "Violência e Abuso Sexual Infantil", orientando para: a detecção de indicadores da situação de violência, fluxo de encaminhamento dos casos através do uso adequado da ficha de notificação disponibilizada pelo município e apresentação dos serviços responsáveis pelo acompanhamento.

Por meio de projeção de slides e coordenação de rodas de conversa, a psicóloga apresentou informações acerca da identificação de sinais de violência e abuso infantil, bem como dedicou escuta atenta ao modo como os diversos profissionais encaminhavam essas questões no cotidiano. Na avaliação final da capacitação, os profissionais envolvidos (inclusive os da área da saúde) relataram que o conteúdo ofertado pela psicóloga era até então desconhecido.

Enquanto efeitos a longo prazo, visou-se colaborar com a promoção de cuidados em saúde infantil e prevenção de violências no território, com enfoque nos seus determinantes e condicionantes sociais, ampliando a compreensão dos diferentes profissionais- psicopedagogas, educadoras infantis, enfermeiro e profissional de educação física.

Ações orientadas para prevenção à saúde também foram introduzidas nas ações de saúde ofertadas pela UBS, envolvendo diferentes integrantes das equipes. Um dos instrumentos utilizados foram os atendimentos clínicos compartilhados com profissionais da eSF e demais residentes; especialmente para atender casos considerados complexos. Baseadas nos preceitos do Apoio Matricial, essas ações conduziram a um regime de coprodução entre profissional de saúde e o apoiador matricial, possibilitando a troca de saberes e de práticas em ato (Campos \& Guarido, 2007; Melo et al., 2012).

Fundamentais para o manejo das demandas direcionadas à psicóloga, as abordagens compartilhadas adotaram como critério o vínculo estabelecido entre os usuários e os profissionais das eSF e aconteciam, geralmente, com agendamento prévio entre os envolvidos no processo.

As consultas de pré-natal compartilhadas com a enfermagem foram direcionadas para gestantes usuárias de drogas e/ou vítimas de violência doméstica- graves problemas de saúde amplamente reconhecidos. Por vezes, profissionais da equipe de enfermagem solicitaram o apoio da psicóloga por não se considerarem capacitados adequadamente quanto ao manejo desse tipo de situação. A inserção da Psicologia na condução e acompanhamento dos casos foi considerada um facilitador pelos profissionais das eSF.

Nessas ocasiões, ao problematizar os valores e crenças que moviam as usuárias nos seus modos de agir e deliberar sobre as próprias vidas, de forma compartilhada com outras áreas do conhecimento científico (Andrade \& Araújo, 2003), observou-se a contribuição da Psicologia para a mudança da abordagem pautada numa concepção estritamente biológica de saúde. Essa dinâmica culminou na corresponsabilização pelo cuidado durante a construção de Projetos Terapêuticos Singulares (PTS): considerando potencialidades e fragilidades, criando 
espaços de reflexão e problematização de aspectos psicossociais intrínsecos às dimensões subjetivas da saúde, do adoecimento e da recuperação (Nepomuceno \& Brandão, 2011).

Essas intervenções foram classificadas como ações de prevenção por terem sido direcionadas a reduzir e evitar o agravamento de problemas específicos de saúde, como a violência intrafamiliar e a dependência química; além do surgimento, a médio e longo prazo, de agravos relativos à gestação de alto risco, como a interrupção da gravidez, depressão pós-parto, morte perinatal e nascimento de bebês com baixo peso e prematuridade (Audi, Segall-Corrêa, Santiago, Andrade, \& Pérez-Escamilla, 2008).

Também, foram realizados grupos voltados à cessação do tabagismo na UBS, vinculados ao Programa Nacional de Controle do Tabagismo (PNCT), os quais foram desenvolvidos em parceria com os residentes de Farmácia e Fisioterapia. Durante a organização e condução dos grupos, observou-se abordagens centradas principalmente no processo bioquímico da dependência, o que dificultava a aproximação dos profissionais junto aos usuários participantes, conforme observado inicialmente pela psicóloga. Desse modo, esta buscou imprimir um olhar compreensivo sobre o hábito de fumar, contribuindo com apoio ao comportamento de cessação do tabagismo, baseando-se na redução de danos para a saúde.

Entende-se que tais intervenções da psicóloga foram fortalecidas a partir da realização de visitas domiciliares, ação expressiva no seu cotidiano de trabalho. Esse recurso pode ser concebido como tecnologia de interação potencialmente capaz de contribuir para uma proposta de atendimento mais compreensiva e vinculada à realidade do usuário.

A abordagem dos usuários inseridos no contexto familiar mostrou-se como um recurso facilitador a fim de trazer à tona os diversos aspectos da realidade social e comunitária da população adscrita, além de propiciar o contato com familiares envolvidos na situação de sofrimento, que também poderiam ser acolhidos e orientados.

As visitas domiciliares compartilhadas com profissionais das eSF e demais residentes ocorreram, muitas vezes, a partir da solicitação por atendimento individual (seja pelos usuários ou pelos profissionais). Como exemplo, tem-se a seguinte situação: um ACS expôs o caso de usuária com queixas de ansiedade, depressão, insônia e isolamento social. Inicialmente, discutiu-se com o ACS e com a profissional residente de Educação Física sobre a estratégia de inserção da usuária nos grupos realizados pelos residentes, por serem considerados recursos de estímulo ao relacionamento interpessoal e manutenção da qualidade de vida.

Em seguida, durante a visita domiciliar com tais profissionais, o enfoque não se restringiu à orientação psicológica, abordando-se também temas como a prática de atividade física, relacionamento familiar e interação social, além dos cuidados com a saúde sexual e reprodutiva.

Tais temas emergiram a partir da problematização dos sintomas relatados pela usuária, associados com as circunstâncias de seu contexto atual: perdas recentes, sedentarismo e ausência de cuidados básicos de saúde. Nesse caso, a abordagem compartilhada favoreceu um olhar ampliado sobre as queixas da usuária, favorecendo uma ação de saúde mais diversificada, que incluiu os cuidados com a saúde quanto a aspectos físicos, mentais e sociais, visando ao estímulo a hábitos saudáveis e extrapolando as queixas psicológicas. Com as ações encaminhadas, almejou-se prevenir agravos específicos na vida da usuária, tais como doenças crônicas e relacionadas ao sistema reprodutor feminino. 
Esse caso ilustra como foi possível, a partir de uma demanda por atendimento clínico individual e fazendo uso de outros recursos, recorrer à prática da Clínica Ampliada extrapolando o manejo restrito aos aspectos psicológicos do sujeito (Campos \& Guarido, 2007), de modo a produzir ações na direção da prevenção à saúde.

Em todas as intervenções discutidas acima, a inserção da psicóloga no trabalho em equipe esteve sensível ao ponto de vista da atividade de trabalho, o que gerava um retrabalho das demandas direcionadas à psicóloga. Adotar essa postura introduzia, no planejamento e execução das ações de saúde, a problematização das escolhas, decisões, normas de cuidados e dos saberes da prática, gerados a partir do que vivem os profissionais de saúde nas situações concretas de trabalho. Acredita-se que esta dinâmica colaborou para enriquecer não apenas o Apoio Matricial, mas, também, para a produção de novos conhecimentos e formas de intervenção.

Ressalta-se que o trabalho compartilhado entre NASF e eSF necessita ser construído e desenvolvido por todos os atores implicados, incluindo-se a população atendida, para além de concepções isoladas e restritas a um campo específico do saber, para que sejam promovidas ações potencializadoras da vida (Perrella, 2015).

\section{Considerações finais}

O desenvolvimento do trabalho na $A B$, via inserção na RMSF, propiciou conhecer a Política Nacional da Atenção Básica (PNAB) e colocá-la em diálogo com a formação específica em Psicologia, ampliando suas possíveis contribuições. Oportunizou refletir e experimentar distintas formas de trabalho na assistência à saúde, à distância de uma perspectiva estritamente individualista e psicopatologizante, orientada exclusivamente para os usuários. Constatou-se que problematizar os saberes práticos dos trabalhadores e adotar o ponto de vista da atividade pode alimentar uma dinâmica adequada para melhor compreender os singulares modos de vida dos usuários e, assim, repensar as ações de saúde ofertadas - como, por exemplo, afastar-se da oferta exclusiva de consultas psicológicas.

O formato organizacional adotado pela RMSF - funcionamento como uma equipe NASF com jornada de trabalho exclusiva em uma única UBS- colaborou para a construção de vínculos não somente com os profissionais da instituição, mas também com profissionais de outros serviços e usuários, além de propiciar o aprofundamento quanto às dinâmicas sociais e comunitárias circulantes no território em questão.

Pautando-se pelas contribuições de Canguilhem, a recepção de usuários conduzidos a partir de queixas ou diagnósticos específicos, não se restringiu à interpretação das causas e efeitos psicológicos. Almejando ampliar a capacidade compreensiva acerca dos modos de vida daqueles e colaborar para o fortalecimento de sua capacidade normativa, articulou-se a escuta qualificada, contextualizando demandas e singularizando-as, por meio de recursos como o acolhimento e o estabelecimento de vínculos.

O trabalho em equipe demonstrou-se fundamental. Em diversos momentos, recorreu-se aos profissionais das eSF fazendo uso das reuniões de matriciamento (espaços formais), assim como de momentos informais para o Apoio Matricial, através de diálogos com trocas de saberes em situações não previamente instituídas.

$O$ trabalho da psicóloga direcionado à promoção e à prevenção à saúde no contexto da $A B$ assinalou para possibilidades de intervenção para além do tradicional atendimento clínico 
individual. No que concerne a essas ações, salienta-se a importância de o psicólogo monitorar, em conjunto com os demais profissionais de saúde, os efeitos dessas ações na vida dos usuários. Desse modo, além de conhecer os hábitos, práticas e modos de viver da população envolvida, podem concretizar propostas que façam sentido em cada realidade social (Andrade \& Araújo, 2003).

Muitas vezes, observou-se indícios de concepções limitadas a respeito dos alcances da Psicologia, tanto por parte de profissionais quanto de usuários. Assim, por meio do diálogo sistemático e respeitoso junto aos diferentes atores implicados, procedeu-se na direção de colaborar para a ampliação do entendimento acerca das possibilidades de contribuição do psicólogo.

\section{Referências}

Amaral, M., Gonçalves, C., \& Serpa, M. (2012). Psicologia comunitária e a saúde pública: Relato de experiência da prática Psi em uma Unidade de Saúde da Família. Psicologia: Ciência e Profissão, 32(2), 484-495. doi: 10.1590/S1414-98932012000200015

Andrade, A. N., \& Araújo, M. D. (2003). Paradoxos das políticas públicas: Programa de saúde da família. In A. N. Andrade, \& Z. A. Trindade (Orgs.), Psicologia e Saúde: Um campo em construção (pp. 73-87). São Paulo: Casa do Psicólogo.

Athayde, M. R. C., \& Zambroni-de-Souza, P. (2015). Por uma ergopsicologia: uma caixa de ferramentas e pistas. In I. M. R. Taveira, A. C. Limongi-França, \& M. C. Ferreira (Orgs.). Qualidade de vida no trabalho: Estudos e metodologias brasileiras (pp. 263-275). Curitiba: CRV.

Audi, C. A. F., Segall-Corrêa, A. M., Santiago, S. M., Andrade, M. G., \& Pérez-Escamilla, R. (2008). Violência doméstica na gravidez: Prevalência e fatores associados. Revista Saúde Pública, 42(5) 877-885. doi: 10.1590/S0034-89102008005000041

Ayres, J. R. C. M., França Junior, I., Calazans, G. J., \& Saletti Filho, H. C. (2003). O conceito de vulnerabilidade e as práticas de saúde: Novas perspectivas e desafios. In D. Czeresnia, \& C. M. Freitas (Orgs.), Promoção da saúde: Conceitos, reflexões, tendências (pp. 117-139). Rio de Janeiro: Fiocruz.

Azevedo, N. S., \& Kind, L. (2013). Psicologia nos Núcleos de Apoio à Saúde da Família em Belo Horizonte. Psicologia: Ciência e Profissão, 33(3), 520-535. doi: 10.1590/ S1414-98932013000300002

Bock, A. M. B. (1999). A Psicologia a caminho do novo século: identidade profissional e compromisso social. Estudos de Psicologia, 4(2), 315-329. doi: 10.1590/ S1413-294X1999000200008

Brasil. (2006). Ministério da Saúde. Portaria no 648, de 28 de março de 2006. Aprova a Política Nacional de Atenção Básica, estabelecendo a revisão de diretrizes e normas para a organização da atenção básica para o Programa Saúde da Família (PSF) e o Programa Agentes Comunitários de Saúde (PACS). Diário Oficial da União, Brasília, DF.

Brasil. (2008). Ministério da Saúde. Portaria no 154, de 25 de janeiro de 2008. Cria os Núcleos de Apoio à Saúde da Família - NASF. Diário Oficial da União, Brasília, DF.

Brasil. (2010). Ministério da Saúde. Diretrizes do NASF: Núcleo de Apoio a Saúde da Família. Secretaria de Atenção à Saúde. Departamento de Atenção Básica (Cadernos de Atenção 
Básica, n. 27). Brasília: Ministério da Saúde. Disponível em http://189.28.128.100/dab/ docs/publicacoes/cadernos_ab/abcad27.pdf

Brasil. (2011). Ministério da Saúde. Portaria no 2.488, de 21 de outubro de 2011. Aprova a Política Nacional de Atenção Básica (PNAB), estabelecendo a revisão de diretrizes e normas para a organização da Atenção Básica, para a Estratégia Saúde da Família (ESF) e o Programa de Agentes Comunitários de Saúde (PACS). Diário Oficial da União, Brasília-DF. Disponível em http://bvsms.saude.gov.br/bvs/saudelegis/gm/2011/ prt2488_21_10_2011.html

Brasil. (2012). Secretaria de Educação Superior. Comissão Nacional de Residência Multiprofissional em Saúde (CNRMS). Resolução no 2, de 13 de abril de 2012. Dispõe sobre Diretrizes Gerais para os Programas de Residência Multiprofissional e em Profissional de Saúde. Diário Oficial da União, Brasília, DF. Disponível em http://portal.mec.gov.br/ index.php?option=com_docman\&view=download\&alias=15448-resol-cnrms-n2-13abril2012\&|temid=30192

Brasil. (2014). Ministério da Saúde. Núcleo de Apoio à Saúde da Família - Volume 1: Ferramentas para a gestão e para o trabalho cotidiano. Secretaria de Atenção à Saúde. Departamento de Atenção Básica (Cadernos de Atenção Básica, n. 39). Brasília: Ministério da Saúde. Disponível em http://bvsms.saude.gov.br/bvs/publicacoes/nucleo_apoio_ saude_familia_cab39.pdf

Campos, G. W. S., \& Cunha, G. T. (2011). Apoio Matricial e Atenção Primária em Saúde. Saúde e Sociedade, 20(4), 961-970. doi: 10.1590/S0104-12902011000400013

Campos, G. W. S., \& Domitti, A. C. (2007). Apoio matricial e equipe de referência: uma metodologia para gestão do trabalho interdisciplinar em saúde. Cadernos de Saúde Pública, 23(2), 399-407. doi: 10.1590/S0102-311X2007000200016

Campos, F. C. B., \& Guarido, E. L. (2007). O psicólogo no SUS: Suas práticas e as necessidades de quem o procura. In M. J. P. Spink (Org.). A psicologia em diálogo com o SUS: Prática profissional e produção acadêmica (pp. 81-103). São Paulo: Casa do Psicólogo.

Caponi, S. (1997). Georges Canguilhem y el estatuto epistemológico del concepto de salud. História, Ciência, Saúde - Manguinhos, 4(2), 287-307. Disponível em http://www.scielo. $\mathrm{br} / \mathrm{pdf} / \mathrm{hcsm} / \mathrm{v} 4 \mathrm{n} 2 / \mathrm{v} 4 \mathrm{n} 2 \mathrm{a005}$.pdf

Cezar, P. K., Rodrigues, P. M. \& Arpini, D. M. (2015). A Psicologia na estratégia de saúde da família: Vivências da residência multiprofissional. Psicologia: Ciência e Profissão, 35(1), 211-224. doi: 10.1590/1982-3703000012014

Clemente, A., Matos, D. R., Grejanin, D. K. M., Santos, H. E. S., Quevedo, M. P., \& Massa, P. A. (2008). Residência multiprofissional em saúde da família e a formação de psicólogos para a atuação na atenção básica. Saúde e Sociedade, 17(1), 176-184. doi: 10.1590/ S0104-12902008000100016

Czeresnia, D. (2003). O conceito de saúde e a diferença entre prevenção e promoção. In D. Czeresnia, \& C. M. Freitas (Orgs.), Promoção da saúde: Conceitos, reflexões, tendências (pp. 1-7). Rio de Janeiro: Fiocruz.

Dimenstein, M. D. (1998). O psicólogo nas unidades básicas de saúde: Desafios para a formação e atuação profissionais. Estudos de Psicologia (Natal), 3(1), 53-81. doi: 10.1590/ S1413-294X1998000100004 
Dimenstein, M. D. (2006). A prática dos psicólogos no Sistema Único de Saúde/SUS. In Conselho Federal de Psicologia [CFP], I Fórum Nacional de Psicologia e Saúde Pública: Contribuições técnicas para avançar o SUS (pp. 08-16). Brasília: CFP. Disponível em https:// pt.scribd.com/document/31815263/I-Forum-Nacional-de-Psicologia-e-Saude-Publicacontribuicoes-tecnicas-e-politicas-para-avancar-o-SUS

Dimenstein, M. D., \& Macedo, J. P. (2007). Desafios para o fortalecimento da Psicologia no SUS: A produção referente à formação e inserção profissional. In M. J. P. Spink (Org.), A psicologia em diálogo com o SUS: Prática profissional e produção acadêmica (pp. 207214). São Paulo: Casa do Psicólogo.

Domingos, C. M., Nunes, E. F. P. A., \& Carvalho, B. G. (2015). Potencialidades da Residência Multiprofissional em Saúde da Família: O olhar do trabalhador de saúde. Interface: Comunicação, Saúde, Educação, 19(55), 1221-1232. doi: 10.1590/1807-57622014.0653

Freitas, C. M. (2003). A vigilância da saúde para a promoção da saúde. In D. Czeresnia, \& C. M. Freitas (Orgs.), Promoção da saúde: Conceitos, reflexões, tendências (pp. 141-159). Rio de Janeiro: Fiocruz.

Macedo, K. B., Heloani, R., \& Cassiolato, R. (2010). O psicólogo como trabalhador assalariado: Setores de inserção, locais, atividades e condições de trabalho. In A. V. B. Bastos, \& S. M. G. Gondim (Orgs.). O trabalho do psicólogo no Brasil (pp.131-150). Porto Alegre: Artmed. Medina, M. G., Aquino, R., Vilasbôas, A. L. Q., Mota, E., Júnior, E. P. P., Luz, L. A., . . Pinto, I. C. M. (2014). Promoção da saúde e prevenção de doenças crônicas: O que fazem as equipes de Saúde da família? Saúde em Debate, 38(número especial), 69-82. doi: 10.5935/0103-1104.2014S006

Melo, L. M., Faria, T. H., Rocha, A. C. P., Oliveira, L. C., Ferraz, D., Araújo, L. R. P., . . Fabbro, M. R. C. (2012). Matriciamento como ferramenta para o processo de trabalho em equipe em uma unidade de saúde da família, Brasil: Relato de experiência. In Ministerio de Salud Pública de Cuba (Org.), Memorias Convención Internacional de Salud Pública "Cuba Salud 2012" (pp. 1-11). La Habana, Cuba. Disponível em http://www.convencionsalud2012.sld. cu/index.php/convencionsalud/2012/paper/viewFile/503/226

Nepomuceno, L. B. (2009). A prática da Psicologia no Nasf. In Conselho Federal de Psicologia, A prática da Psicologia e o Núcleo de Apoio à Saúde da Família (pp. 49-59). Brasília: CFP. Disponível em http://site.cfp.org.br/wp-content/uploads/2009/12/Seminxrio_O_Nxcleo_ de_Apoio-beta.pdf

Nepomuceno, L. B., \& Brandão, I. R. (2011). Psicólogos na Estratégia Saúde da Família: Caminhos percorridos e desafios a superar. Psicologia: Ciência e Profissão, 31(4), 762-777. doi: 10.1590/S1414-98932011000400008

Oliveira, S. S., \& Brito, J. C. (2011). A dimensão gestionária do trabalho e o debate de normas e valores no teleatendimento. Trabalho, Educação e Saúde, 9(1), 265-284. doi: 10.1590/ S1981-77462011000400013

Perrella, A. C. (2015). A experiência da Psicologia no NASF: Capturas, embates e invenções. Gerais: Revista Interinstitucional de Psicologia, 8(2), 443-452. Disponível em http://pepsic. bvsalud.org/scielo.php?script=sci_abstract\&pid=\$1983-82202015000300012

Sícoli, J. L., \& Nascimento, P. R. (2003). Promoção de saúde: concepções, princípios e operacionalização. Interface: Comunicação, Saúde, Educação, 7(12), 91-112. doi: 10.1590/ S1414-32832003000100008 
Spink, M. J., Menegon, V. S. M., Gamba, E. A. C., \& Lisboa, M. S. (2007). Contribuições da Psicologia para a saúde pública: Onde publicamos, a quem endereçamos e que efeitos podemos ter. In M. J. P. Spink (Org.), A psicologia em diálogo com o SUS: Prática profissional e produção acadêmica (pp. 141-174). São Paulo: Casa do Psicólogo.

Spink, M. J. P. (2003). Psicologia social e saúde: Práticas, saberes e sentidos. Petrópolis, RJ: Vozes.

Recebido em: 28/11/2017

Última revisão: $12 / 07 / 2018$

Aceite Final: 09/10/2018

\section{Sobre as autoras:}

Natália Batista Rosa - Especialista em Saúde da Família - área de concentração em Psicologia - pelo Programa de Residência Multiprofissional em Saúde da Família da Universidade Estadual de Londrina (UEL). Especialista em Saúde Coletiva e Saúde da Família pelo Centro Universitário Filadélfia (UniFil). Psicóloga pela UEL. Psicóloga clínica e tutora das disciplinas interativas dos cursos de graduação presencial em Psicologia, na Kroton Educacional. E-mail: nataliab.rosa@hotmail.com, Orcid: http://orcid. org/0000-0003-4098-9768

Ana Cláudia Barbosa da Silva-Roosli - Doutora em Psicologia Social pela Universidade do Estado do Rio de Janeiro (UERJ), Brasil. Docente do Departamento de Psicologia Social e Institucional da Universidade Estadual de Londrina (UEL). Brasil. E-mail: acbs79@gmail.com, Orcid: http://orcid.org/0000-0003-3795-9357 